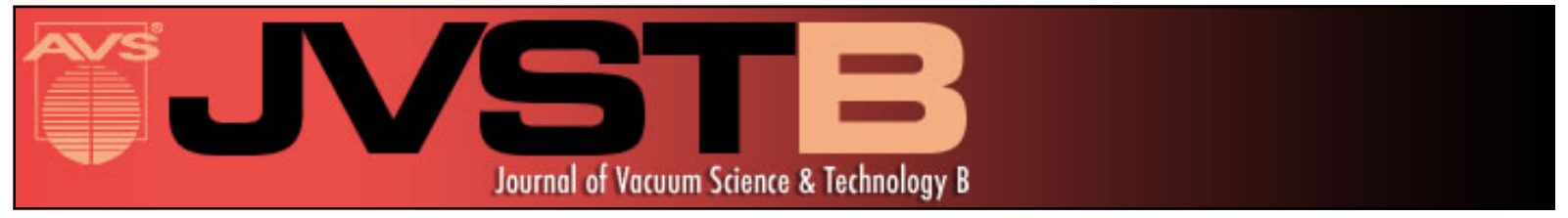

\title{
Long wavelength pseudomorphic InGaPAsSb type-I and type-II active layers grown on
} GaAs

S. R. Johnson, P. Dowd, W. Braun, U. Koelle, C. M. Ryu, M. Beaudoin, C.-Z. Guo, and Y.-H. Zhang

Citation: Journal of Vacuum Science \& Technology B 18, 1545 (2000); doi: 10.1116/1.591423

View online: http://dx.doi.org/10.1116/1.591423

View Table of Contents: http://scitation.aip.org/content/avs/journal/jvstb/18/3?ver=pdfcov

Published by the AVS: Science \& Technology of Materials, Interfaces, and Processing

\section{Articles you may be interested in}

Highly tensile-strained, type-II, Ga $1-\mathrm{x} \ln \mathrm{x}$ As / GaSb quantum wells

Appl. Phys. Lett. 96, 062109 (2010); 10.1063/1.3303821

GaAs-substrate-based long-wave active materials with type-II band alignments

J. Vac. Sci. Technol. B 19, 1501 (2001); 10.1116/1.1386380

Strained InGaAs/GaPAsSb heterostructures grown on GaAs (001) for optoelectronic applications in the $1100-1550 \mathrm{~nm}$ range

J. Appl. Phys. 88, 3004 (2000); 10.1063/1.1287233

Observation of direct (type-I) transitions in type-II InGaAs/AIAsSb heterostructures lattice matched to InP grown by molecular beam epitaxy

J. Vac. Sci. Technol. B 18, 1586 (2000); 10.1116/1.591432

Molecular beam epitaxial growth of $\operatorname{lnGaAsN}: \mathrm{Sb} / \mathrm{GaAs}$ quantum wells for long-wavelength semiconductor lasers Appl. Phys. Lett. 75, 178 (1999); 10.1063/1.124311

\section{SHIMADZU Powerful, Multi-functional UV-Vis-NIR and Excellence in Science FTIR Spectrophotometers}

Providing the utmost in sensitivity, accuracy and resolution for applications in materials characterization and nano research

- Photovoltaics

- Polymers

- Thin films

- Paints

- Ceramics

- DNA film structures

- Coatings

- Packaging materials

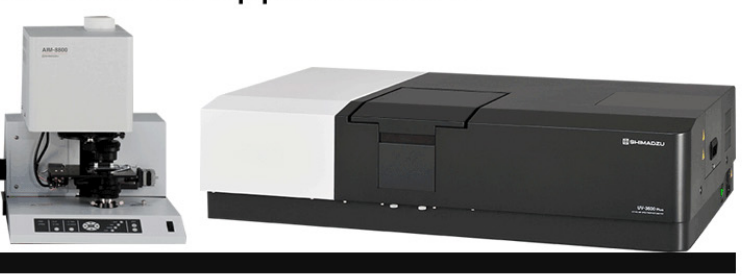




\title{
Long wavelength pseudomorphic InGaPAsSb type-I and type-II active layers grown on GaAs
}

\author{
S. R. Johnson, a) P. Dowd, W. Braun, U. Koelle, C. M. Ryu, M. Beaudoin, C.-Z. Guo, \\ and Y.-H. Zhang \\ Center for Solid State Electronics Research, Department of Electrical Engineering, \\ Arizona State University, Tempe, Arizona 85287-6206
}

(Received 10 October 1999; accepted 14 January 2000)

\begin{abstract}
GaPAsSb type-I quantum wells and symmetric InGaAs/GaPAsSb/InGaAs type-II quantum wells are proposed as long wavelength active layers for GaAs based optoelectronic devices. Room temperature photoluminescence from 1.2 to $1.5 \mu \mathrm{m}$ is observed from the type-II quantum wells, while low temperature photoluminescence at $1.25 \mu \mathrm{m}$ is observed from type-I quantum wells. A reflection high-energy electron diffraction peak that is extended parallel to the surface, while its width is perpendicular to the surface is narrow, suggests that spontaneous lateral composition modulation occurs in the GaPAsSb layers. (C) 2000 American Vacuum Society.
\end{abstract}

[S0734-211X(00)02703-7]

\section{INTRODUCTION}

Vertical-cavity surface-emitting lasers (VCSELs) operating at 1.3 and $1.55 \mu \mathrm{m}$ are desirable for a wide range of applications, such as optical communication and optical interconnects. The development of optical interconnects is important for many applications such as image processing in synthetic aperture radar, real-time tasking of multisensor platforms, dynamic target recognition, virtual reality systems, rapid searches of extremely large dynamic databases, and local area networks. Long wavelength VCSELs that operate at high data rates are anticipated to be key components in the development of optical interconnects, gigabit Ethernet, fiber to the home, and optical local area networks.

Hybrid integration of VCSELs and photodetectors with $\mathrm{Si}$ circuitry is a promising low-cost technology for future highspeed free-space optical interconnects. Because of the ever decreasing operating voltages in Si integrated circuits (ICs), longer wavelength (i.e., lower operating voltage) VCSELs will soon be required for integrated optical interconnects. In a few years Si IC voltages will be below the threshold voltages of the 980 and $850 \mathrm{~nm}$ lasers that are now commonly used in short distance optical communication. Therefore, the development of longer wavelength (smaller band gap) materials for short distant optical links is critical. The logical choices for these longer wavelengths are 1.3 and $1.55 \mu \mathrm{m}$ as these wavelengths are already extensively used in longer haul optical communications.

There has been considerable interest in developing long wavelength VCSELs using a number of different approaches based on InP and GaAs substrates. Fabrication of VCSELs using the InP/InGaAsP system has several drawbacks when compared to the GaAs based system. For example, both refractive index contrast and thermal conductivity are superior in the GaAs based system. ${ }^{1}$ These two attributes are important for the fabrication of suitable distributed Bragg reflector (DBR) mirrors for VCSELs. A complex approach that over-

${ }^{a)}$ Electronic mail: shane.johnson@asu.edu comes the limitation of InP based DBRs is wafer fusion, where InP based active regions are bonded to GaAs based DBRs. ${ }^{2}$

It can be difficult to find materials with band gaps suitable for $1.3 \mu \mathrm{m}$ emission that can be grown on GaAs. In one approach, quantum dot (QD) structures have been developed that produce photoluminescence (PL) at up to $1.3 \mu \mathrm{m} .{ }^{3}$ More recently, $1.31 \mu \mathrm{m}$ edge-emitting QD lasers have been demonstrated, ${ }^{4}$ where long $(2.1 \mathrm{~mm})$ cavity and high reflectivity facet coating were required to achieve lasing. Room temperature operation of QD-based VCSELs has also been achieved, ${ }^{5}$ but the lasing wavelength was only $1.15 \mu \mathrm{m}$ PL at wavelengths of up to $1.3 \mu \mathrm{m}$ has also been observed in $\mathrm{GaAsSb} / \mathrm{InGaAs}$ bilayer type-II quantum well (QW) samples. ${ }^{6}$ To date, the longest emission wavelength in GaAsbased VCSEL structures is with a single step growth 1.18 $\mu \mathrm{m}$, using a single InGaNAs QW. ${ }^{7}$ Though it is difficult to incorporate significant levels of $\mathrm{N}$ in InGaAs, a $1.3 \mu \mathrm{m}$ edge-emitting laser with an InGaNAs QW (containing $1 \%$ N) has been realized. ${ }^{8}$

To overcome the limitations of InP based DBRs and wafer bonding, and because GaAs is in general a more mature technology, we are developing novel long-wavelength type-I and type-II InGaPAsSb QW systems for the GaAs system. These structures are grown coherently strained on GaAs by solid source molecular beam epitaxy (MBE). We propose $\mathrm{In}_{x} \mathrm{Ga}_{1-x} \mathrm{P}_{y} \mathrm{As}_{z} \mathrm{Sb}_{1-y-z}$ type-I QWs $(0.00 \leqslant x \leqslant 0.04$, $0.3 \leqslant y \leqslant 0.5,0.0 \leqslant z \leqslant 0.2$ ) for wavelengths up to $1.3 \mu \mathrm{m}$ and symmetric type-II $\quad \mathrm{In}_{x} \mathrm{Ga}_{1-x} \mathrm{As}_{2} / \mathrm{GaP}_{y} \mathrm{As}_{z} \mathrm{Sb}_{1-y-z}$ / $\operatorname{In}_{x} \mathrm{Ga}_{1-x}$ As QWs $(0.3 \leqslant x \leqslant 0.4,0.1 \leqslant y \leqslant 0.5,0.0 \leqslant z \leqslant 0.5)$ for wavelengths up to $1.55 \mu \mathrm{m}$. Preliminary results indicate that 1.3 and $1.55 \mu \mathrm{m}(0.95$ to $0.80 \mathrm{eV})$ wavelengths can be reached without exceeding 3\% compressive strain levels.

The samples presented here were grown using a VG V80H solid source MBE system equipped with As, $\mathrm{P}$, and $\mathrm{Sb}$ group-V valved crackers, $\mathrm{Ga}$, In, and $\mathrm{Al}$ group-III effusion cells, Si and GaTe cells for $n$-type doping, and a Be cell for $p$-type doping. This MBE system is outfitted with a $15 \mathrm{keV}$ 


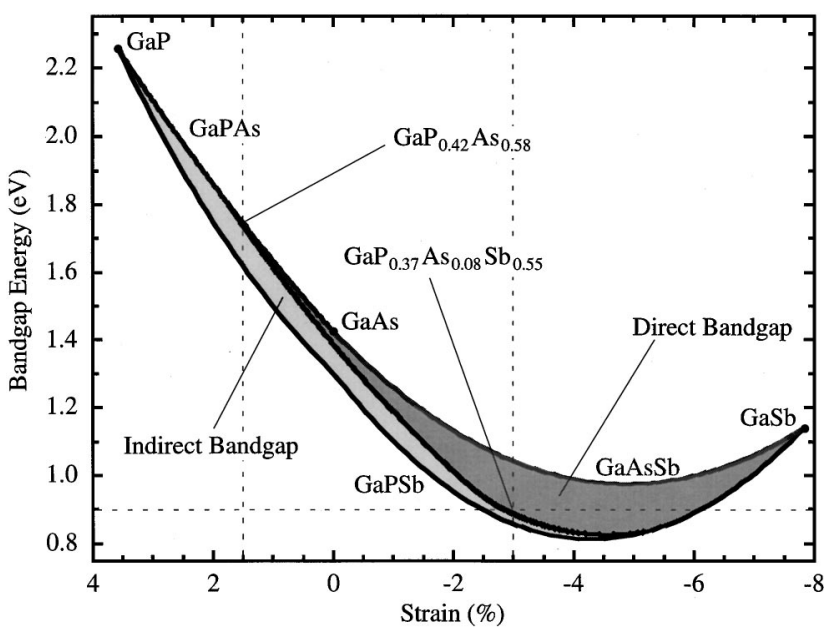

FIG. 1. $\Gamma$ point band gap energy vs strain for coherently strained GaPAsSb on GaAs. Negative strain values indicate compressive strain. The right-hand shaded region is direct band gap material while the left-hand shaded region is indirect band gap material.

reflection high-energy electron diffraction (RHEED) system with a charge coupled device camera and software for realtime acquisition of RHEED data. This RHEED system has the ability to phase-lock (or synchronize) the data acquisition to substrate rotation, ${ }^{9}$ which allows for several real-time observation modes during substrate rotation. The control of the group-V element ratio can be problematic when incorporating three group- $\mathrm{V}$ materials in a single layer. RHEED is one of the key tools used to sort out the conditions required for the optimum growth of these structures.

\section{TYPE-I QUANTUM WELLS}

Though not obvious, because $\mathrm{GaP}$ is an indirect band gap material with a band gap greater than $2 \mathrm{eV}$, emission wavelengths up to $1.3 \mu \mathrm{m}$ are achievable using pseudomorphic (In)GaPAsSb type-I QWs on GaAs. This is mainly due to the large band gap bowing parameter of GaPSb. Furthermore, the small size of the $\mathrm{P}$ atom helps to reduce the strain in the material. However, it is not possible to use GaPSb by itself because it is an indirect band gap material for the strain levels required for a two-dimensional growth mode in MBE, i.e., less than about $3 \%$ strain. The novelty of this approach is that the addition of a small amount of As or InAs to $\mathrm{GaPSb}$ results in a suitable direct band gap active material for wavelengths up to $1.3 \mu \mathrm{m}$. The attributes of this system that make it suitable for GaAs-based lasers are the large bowing band gap parameter of GaPSb and the large range of mixed P, As, and Sb compositions where (In)GaPAsSb is a direct band gap material.

The $\Gamma$ point band gap energy versus strain for coherently strained GaPAsSb on GaAs is shown in Fig. 1. (Note that in Fig. 1 the ternaries GaPAs, GaPSb, and GaAsSb ternaries border the GaPAsSb quaternary region.) In the region labeled "direct band gap", the lowest energy band to band transition is between the $\Gamma$ point of the conduction band and the $\Gamma$ point of the valence band. These direct band gap com-

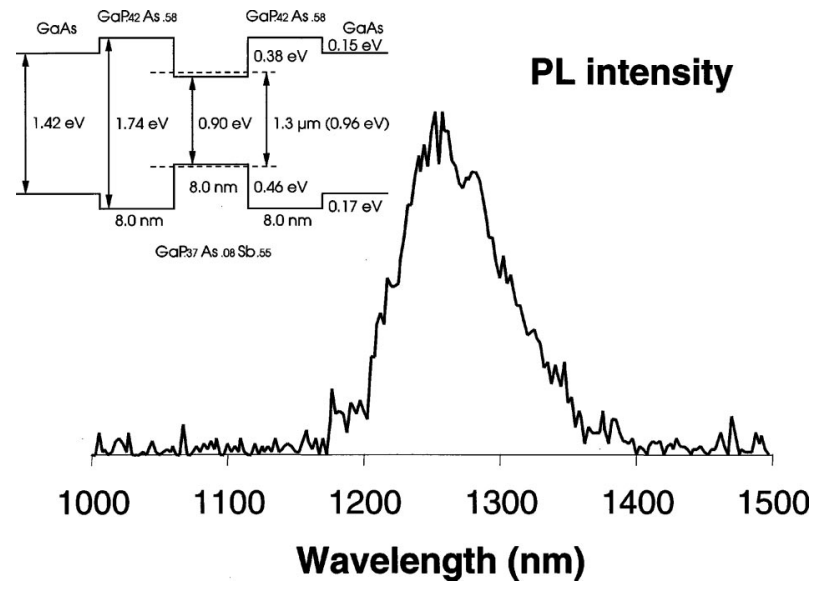

FIG. 2. Low temperature photoluminescence spectrum from a strain compensated GaPAsSb type-I quantum well grown on GaAs. A band diagram of the type-I quantum well is shown in the inset. The $8 \mathrm{~nm}$ GaPAs spacers are $1.5 \%$ tensilely strained while the $8 \mathrm{~nm}$ GaPAsSb QW is $3 \%$ compressively strained.

positions are suitable as active materials for lasers. In the region labeled "indirect band gap", the lowest energy band to band transition is an indirect transition that occurs between either the $X$ or the $L$ valley of the conduction band and the $\Gamma$ point of the valence band. These compositions are not suitable as active materials for lasers. In the indirect band gap region, the lowest energy point of the conduction band occurs at the $L$ valley for compositions nearer $\mathrm{GaSb}$ and at the $X$ valley for compositions nearer GaP.

Most of the bowing parameters used in the calculations for Fig. 1 were obtained from the literature. However, since no data is available for the $L$ valley bowing parameter of $\mathrm{GaPSb}$, the $X$ valley value was used in its place. Measurements of the GaPSb material system indicate that the band gap bowing parameters for this material are very large, 3.8 $\mathrm{eV}$ at the $\Gamma$ point and $2.7 \mathrm{eV}$ for the $X$ valley. ${ }^{10}$ The bowing parameters for GaPAs and GaAsSb are much smaller than those for GaPSb and were obtained from Adachi, Refs. 11 and 12, respectively. Finally Vegard's Law is assumed in the strain calculations, i.e., strain is a linear function of mole fraction.

A schematic band diagram of a strain compensated 1.3 $\mu \mathrm{m}$ GaPAsSb type-I QW is shown in the inset of Fig. 2. The $8 \mathrm{~nm}$ GaPAs spacers are $1.5 \%$ tensilely strained and the 8 $\mathrm{nm} \mathrm{GaP}_{0.37} \mathrm{As}_{0.08} \mathrm{Sb}_{0.55} \mathrm{QW}$ is $3 \%$ compressively strained. The use of strain compensation layers permits the growth of multiple unrelaxed QWs that are necessary for the fabrication of VCSELs. Figure 2 shows preliminary low temperature $(4 \mathrm{~K}) \mathrm{PL}$ results for the strain compensated type-I QW (nominally shown in the inset). The excitation density was $250 \mathrm{~W} / \mathrm{cm}^{2}$ and the peak of the spectrum occurred at 1.25 $\mu \mathrm{m}$. Though room temperature PL was not observed from this sample an emission wavelength of about $1.3 \mu \mathrm{m}$ is expected at room temperature when the temperature dependence of the band gap is considered. This result indicates that the $\mathrm{GaP}_{0.37} \mathrm{As}_{0.08} \mathrm{Sb}_{0.55}$ is a good candidate for a $1.3 \mu \mathrm{m}$ type-I active material. 


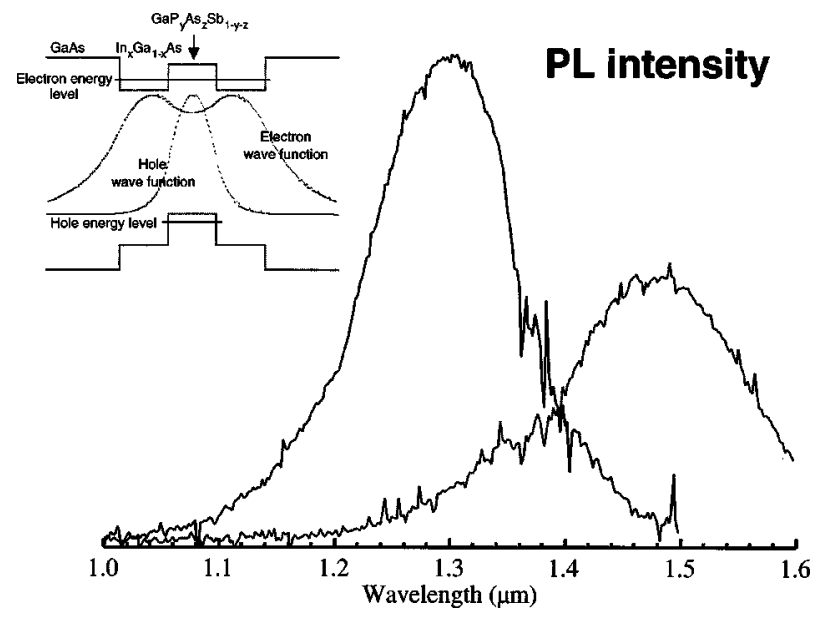

FIG. 3. Room temperature photoluminescence spectra from InGaAs/ GaPAsSb/InGaAs type-II quantum wells grown on GaAs. Varying the composition of the GaPAsSb layer changes the emission wavelength. Each layer is nominally $3 \%$ compressively strained. A band diagram and the electron and hole wave function of the type-II quantum well are shown in the inset.

\section{TYPE-II QUANTUM WELLS}

A type-II QW is formed using two semiconductor materials that have a staggered band alignment, where the conduction band and valence band edges of one semiconductor are both shifted in the same direction relative to the band edges of the other semiconductor. In the classical picture, the resulting optical transition in such a structure is perceived as being spatially indirect because the electrons (holes) are thought to reside in lowest (highest) part of the well. However, in the more accurate quantum picture where the electron and hole wave functions are considered, the transition is direct in the sense that it is the spatial overlap of the wave functions that is important. With this system a smaller effective band gap (optical transition) is obtained at the cost of wave function overlap. The idea to use a type-II heterojunction for infrared emission was first proposed by Kroemer and Griffiths. ${ }^{13}$

In the case of symmetric type-II QWs, a smaller effective band gap is achieved at the cost of a broader electron wave function and a narrower hole wave function (see Fig. 3 inset). While for asymmetric type-II QWs the electron and hole wave functions are spatially offset. In both cases, this results in a reduction of the electron-hole wave function overlap, which reduces the oscillator strength when compared to type-I QWs. However, theoretical calculations show that in spite of the reduced wave function overlap, the gain levels required for lasers are attainable in symmetric type-II QWs. ${ }^{14}$ For an $\mathrm{In}_{0.4} \mathrm{Ga}_{0.6} \mathrm{As} / \mathrm{GaP}_{0.15} \mathrm{As}_{0.40} \mathrm{Sb}_{0.45} / \mathrm{In}_{0.4} \mathrm{Ga}_{0.6} \mathrm{As}$ (2.3 nm-2.3 nm-2.3 nm) QW structure embedded in GaAs, the calculated transition wavelength is close to $1.3 \mu \mathrm{m}$ and the electron-hole wave function overlap is better than $65 \%$. Such a large electron-hole wave function overlap is the foundation of the symmetric type-II QW design. A peak gain of $10^{4} \mathrm{~cm}^{-1}$ is predicted for a carrier density of $10^{19} \mathrm{~cm}^{-3},{ }^{14}$ which is not unreasonable for the operation of VCSELs.

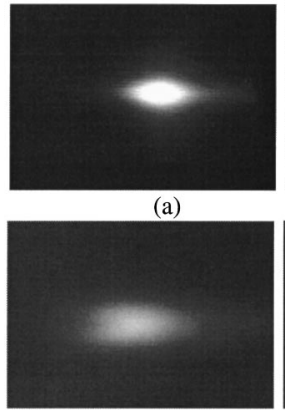

(d)

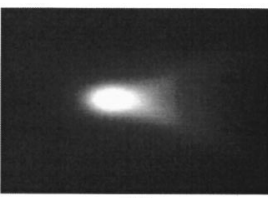

(b)

(e)

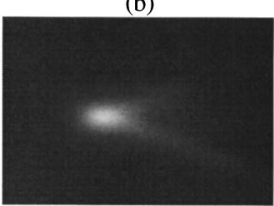

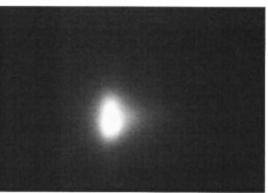

(c)

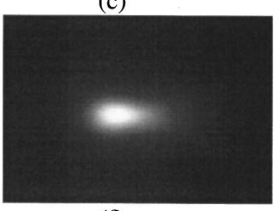

FIG. 4. Reflection high-energy electron diffraction images taken through the growth cycle of a symmetric InGaAs/GaPAsSb/InGaAs type-II quantum well: (a) GaAs surface prior to first InGaAs layer, (b) end of the first InGaAs layer, (c) central GaPAsSb layer, (d) start of second InGaAs layer, (e) end of second InGaAs layer and (f) start of GaAs overgrowth.

A schematic of the band-edge alignment and the electron and hole wave functions of the proposed type-II QWs is shown in the inset of Fig. 3. The band offsets have been calculated using the model-solid theory ${ }^{15}$ and the effects of strain have been included. Though not shown, GaPAs strain compensation layers can also be used to extend this system to multiple QWs.

Two symmetric type-II QWs samples (A and B) with different compositions for the center GaPAsSb layer were grown at $505{ }^{\circ} \mathrm{C}$ on semi-insulating GaAs. Each individual InGaAs and GaPAsSb layer thickness and strain are estimated to be $3 \mathrm{~nm}$ and $3 \%$, respectively. Depending on the composition of the central GaPAsSb layer, the peak of the emission spectrum can be shifted from 1.2 to $1.5 \mu \mathrm{m}$ (see Fig. 3). Increasing the $\mathrm{Sb}$ to $\mathrm{P}$ flux ratio shifts the emission spectrum to longer wavelengths. Theoretical modeling shows that these extended wavelengths are attributed to a symmetric type-II QW.

Figure 3 shows the PL spectra for the two samples studied. For sample A, the peak of the PL spectrum is at $1.3 \mu \mathrm{m}$ with a full-width at half-maximum (FWHM) of $110 \mathrm{meV}$. For sample B, the peak wavelength is close to $1.5 \mu \mathrm{m}$ with a FWHM of $130 \mathrm{meV}$. These broad linewidths (in the type-II structures) are believed to be due to band filling, ${ }^{16}$ fluctuations in the well width and composition, ${ }^{17}$ and InGaAs well asymmetry due to indium segregation in the growth direction.

The inhomogeneous contribution to the broadening of the linewidth in Fig. 3 is due to a Stranski-Krastanov transition that occurs very close to the completion of growth of each InGaAs layer [see RHEED images Figs. 4(a)-4(b) and 4(d)4(e)]. High-resolution secondary ion mass spectrometry measurements indicate that the indium is segregating, and is therefore present in the GaPAsSb layer, while $\mathrm{P}$ and $\mathrm{Sb}$ incorporate in their intended position. In addition to the ratios of the three group- $\mathrm{V}$ elements, the properties of the GaPAsSb layer strongly depend on the indium segregation. The first InGaAs layer forms a rough surface on which the GaPAsSb layer deposits preferentially smoothing the growth 
surface [see Fig. 4(c)] before the second InGaAs layer is deposited.

RHEED results indicate that the surface is disordered when the group- $\mathrm{V}$ fluxes are switched back to solely arsenic after the growth of the GaPAsSb layer [see Figs. 4(c) and 4(d)]. Surface order can be inferred from the intensity of the RHEED patterns, where a well ordered surface results in sharp, intense diffraction peaks with a low background [see Fig. 4(c)] and a disordered surface results in a low intensity diffraction peak [see Fig. 4(d)]. Furthermore during GaPAsSb deposition, the diffraction peak is extended parallel to the surface, while its width perpendicular to the surface is narrow [see Fig. 4(c)].

This unusual shape can be explained by a lateral composition modulation in the growing layer. The different groupIII and group-V elements involved are each from different periods of the periodic table, resulting in strong scattering contrast. Both a lateral phase separation of the GaPAsSb with homogeneous layer thickness due to strain modulation by the InGaAs template or deposition of homogeneous GaPAsSb between the InGaAs mounds with exposed InGaAs could lead to such a modulation. A combination of these two growth modes is also possible. Such a modulation with a periodicity around $40 \mathrm{~nm}$ would lead to a diffraction spot broadening of about $1 \%$ of the Brillouin zone. This agrees with the observed spot size.

The layer structure of the type-II QW samples was examined using cross-sectional transmission electron microscopy. ${ }^{18}$ Lattice imaging showed no visible sign of structural defects. However, the electron micrographs of sample B showed in plane undulating contrast within the QW structure, confirming the thickness and composition variations inferred from the RHEED measurements.

Though these material systems sound very promising there are several growth issues that must be solved to ensure that high quality optoelectronic materials can be grown. Apart from problems like strain relaxation and interface segregation, the role of possible spontaneous lateral composition modulation warrants further study.

\section{CONCLUSIONS}

Long wavelength GaPAsSb type-I and InGaAs/GaPAsSb type-II active materials for GaAs based optoelectronic de- vices are proposed. Low temperature photoluminescence emission at $1.25 \mu \mathrm{m}$ is observed from the type-I GaPAsSb quantum well. Room temperature photoluminescence at 1.2$1.5 \mu \mathrm{m}$ is observed from the type-II quantum wells; increasing the $\mathrm{Sb}$ to $\mathrm{P}$ ratio in the GaPAsSb layer extends the emission wavelength. During GaPAsSb deposition, the reflection high-energy electron diffraction peak is extended parallel to the surface, while its width perpendicular to the surface is narrow, indicating that spontaneous lateral composition modulation occurs.

${ }^{1}$ J. Piprek and S. J. B. Yoo, Electron. Lett. 30, 866 (1994).

${ }^{2}$ J. J. Dudley, D. I. Babic, L. Yang, B. I. Miller, R. J. Ram, T. Reynolds, E. L. Hu, and J. E. Bowers, Proc. LEOS 93, IEEE Lasers and Electro-Optics Society 1993 Annual Meeting, p. 560.

${ }^{3}$ R. P. Mirin, J. P. Ibbetson, K. Nishi, A. C. Gossard, and J. E. Bowers, Appl. Phys. Lett. 67, 3795 (1995).

${ }^{4}$ D. L. Huffaker, G. Park, Z. Zou, O. B. Shchekin, and D. G. Deppe, Appl. Phys. Lett. 73, 2564 (1998).

${ }^{5}$ M. Yamada, T. Anan, K. Tokutome, K. Nishi, A. Gomyo, and S. Sugou, Proc. LEOS 98, IEEE Lasers and Electro-Optics Society 1998 Annual Meeting, p. 149.

${ }^{6}$ M. Peter, K. Winkler, M. Maier, N. Herres, J. Wagner, D. Fekete, K. H. Bachem, and D. Richards, Appl. Phys. Lett. 67, 2639 (1995).

${ }^{7}$ M. C. Larson, M. Kondow, T. Kitatani, K. Nakahara, K. Tamura, H. Inoue, and K. Uomi, IEEE Photonics Technol. Lett. 10, 188 (1988).

${ }^{8}$ K. Nakahara, M. Kondow, T. Kitatani, M. C. Larson, and K. Uomi, IEEE Photonics Technol. Lett. 10, 487 (1998).

${ }^{9}$ W. Braun, H. Möller, S. R. Johnson, and H.-Y. Zhang, J. Vac. Sci. Technol. B 17, 474 (1999).

${ }^{10}$ M. J. Jou, Y. T. Cheng, H. R. Jen, and G. B. Stringfellow, J. Cryst. Growth 93, 62 (1988).

${ }^{11} \mathrm{~S}$. Adachi, Physical Propeties of III-V Semiconductor Compounds (Wiley, New York, 1992).

${ }^{12}$ S. Adachi, J. Appl. Phys. 61, 4869 (1987).

${ }^{13}$ H. Kroemer and G. Griffiths, IEEE Electron Device Lett. EDL-4, 20 (1983).

${ }^{14}$ C. Z. Guo, S. L. Chen, P. Dowd, and Y.-H. Zhang, Appl. Phys. Lett. (submitted).

${ }^{15}$ C. G. Van de Walle, Phys. Rev. B 39, 1871 (1989).

${ }^{16}$ Y.-H. Zhang, R. H. Miles, and D. H. Chow, IEEE J. Sel. Top. Quantum Electron. 1, 749 (1995)

${ }^{17}$ A. Chin and W. J. Chen, Appl. Phys. Lett. 69, 443 (1996).

${ }^{18}$ P. Dowd, W. Braun, D. J. Smith, C. M. Ryu, C.-Z. Guo, S. L. Chen, U. Koelle, S. R. Johnson, and Y.-H. Zhang, Appl. Phys. Lett. 75, 1267 (1999). 